\title{
Older age onset rheumatoid arthritis with or without osteoarthritis
}

\author{
KOJI INOUE, KANJI SHICHIKAWA, JUNICHI NISHIOKA, AND \\ SHIGENAGA HIROTA
}

From the Department of Orthopaedic Surgery, Shiga University of Medical Science, Otsu, Japan

SUMmARY The clinical features of a group of 79 patients with older age onset rheumatoid $\frac{\infty}{2}$ arthritis (ORA) were compared with those of a group of 414 patients with younger age onset $\vec{\circ}$ rheumatoid arthritis. The ORA group contained approximately equal numbers of men and $\vec{N}$ women, were less rheumatoid factor positive, had a raised erythrocyte sedimentation rate, lower HLA-DR4 positivity, and a tendency towards larger joint involvement at the onset of the $\infty$ disease. These features have been reported by many authors except for the lower DR4 positivity. ㅇ Of these features, the lower prevalence of rheumatoid factor positivity and the tendency towards larger joint involvement at the onset were characteristic of a subset of patients with ORA who had had osteoarthritis before the onset of rheumatoid arthritis. It is suggested that ostecarthritic $\Phi$ large joints may be susceptible to the occurrence of rheumatoid synovitis at the onset of the disease, but that the osteoarthritis inducing factor may be negatively related to the progression of $\stackrel{\mathscr{Q}}{=}$ rheumatoid arthritis.

Key words: rheumatoid factor, HLA typing, large joint involvement.

The characteristic clinical features of older age onset rheumatoid arthritis (ORA) have been described by many authors. ${ }^{1-13}$ The data in previous reports were discordant in some respects, but equal distribution of the disease between men and women, a tendency towards large joint involvement, an increased erythrocyte sedimentation rate (ESR), less seropositivity, a stormy onset, and a good prognosis have been mentioned as characteristic manifestations of ORA.

It is supposed, on the other hand, that degenerative joint disease, widely seen in this age group, may have an influence on the clinical manifestations of rheumatoid arthritis (RA), contributing to the clinical features of ORA.

We examined the following parameters in a group of patients with ORA:distribution between the sexes, prevalence of seropositivity, ESR, white blood cell (WBC) count, joints involved at the onset, and HLA typing, and compared these features with those of a group of patients with younger age onset rheumatoid arthritis (YRA).

Accepted for publication 23 May 1987.

Correspondence to Dr Koji Inoue, Department of Orthopaedic Surgery, Shiga University of Medical Science, Otsu, Shiga 520-21, Japan.
Each of above clinical findings was also examined in two subsets of the ORA group, i.e., ORA with osteoarthritis at the onset of RA and ORA without it. The modification of the clinical features of RA by concomitant osteoarthritis is discussed.

\section{Patients and methods}

Patients with ORA were defined as those in whom the disease began after their 60th birthday and patients with YRA as those whose disease occurred at an earlier age as has been described by Terkeltaub et al. ${ }^{12}$ Seventy nine patients with ORA and 414 patients with YRA satisfying the American Rheu- $\frac{O}{2}$ matism Association criteria for definite or classical $D$ RA were investigated. All patients who had attended the orthopaedic outpatient clinic of Shiga University $\mathcal{O}^{\circ}$ of Medical Science during the preceding seven years were investigated.

The values of the ESR and WBC count were $\omega$ those obtained at the first visit. The ESR was? measured by the Westergren method. RF sero- $\varrho$ positivity was determined with the latex fixation $\Phi$ test. Patients were considered seropositive if their $\stackrel{?}{?}$ latex titre was greater than or equal to $1 / 80$.

HLA-A, B, and C antigens were defined by the standard National Institute of Health technique. 
HLA-DR antigens were defined with $B$ lymphocytes isolated from the peripheral blood by their adherence to nylon wool columns. Local and International Workshop sera were used to detect all HLA-A, B, C. and DR specificities.

For the convenience of assessing osteoarthritis, roentgenograms of either the fingers or the knees were used. Osteoarthritis was defined as present when there was a change of grade 2 or more according to the criteria of Kellgren and Lawrence. ${ }^{14}$

Radiological examinations of the hand and knee were available at the first visit for 64 patients with ORA, of whom 33 had osteoarthritis (ORA with OA) and 31 did not (ORA without OA). The distribution between the sexes, prevalence of RF positivity, ESR, prevalence of HLA-DR4 positivity, and involvement of the knees or shoulders at the onset were compared between the two subsets.

Radiological examinations of the knee joints at the onset of RA were available for 51 patients with ORA, in whom the initial joint manifestation was in the knee for 17 patients and in other joints for 34 patients. The prevalence of gonarthrosis was compared between the two groups.

The results were analysed by $\chi^{2}$ test and by Student's $t$ test.

\section{Results}

Comparisons of clinical parameters in ORA and YRA are presented in Tables 1,2 , and 3 . The male:female ratio was $1: 1.4$ in ORA and $1: 3.5$ in YRA (Table 1); the difference was significant $(p<0 \cdot 005)$. Serum RF positivity was lower in ORA $(66 \%)$ than in YRA (76\%; Table 1). The significance of this difference was borderline $(p=0.06)$. The average ESR was $65 \mathrm{~mm} / \mathrm{h}$ in ORA and 46 $\mathrm{mm} / \mathrm{h}$ in YRA (Table 1 ); the difference was highly significant $(p<0.005)$. There were no significant differences in the mean WBC count.

Data on HLA typing were available for 27 patients with ORA and 80 with YRA. The positivity (\%) of selected HLA-A, C, and DR antigens is listed in Table 2. Although no statistical differences

Table 1 Comparison of sex ratio and laboratory data in patients with $O R A$ and $Y R A$

\begin{tabular}{|c|c|c|c|}
\hline & $\begin{array}{l}\text { ORA } \\
(n=79)\end{array}$ & $\begin{array}{l}Y R A \\
(n=414)\end{array}$ & Significance \\
\hline $\begin{array}{l}\text { Sex ratio (M:F) } \\
\text { RF positivity (\%) } \\
\text { ESR (average) } \\
\quad(\mathrm{mm} / \mathrm{h}) \\
\mathrm{WBCl} \text { (average) }\end{array}$ & $\begin{array}{l}1: 1 \cdot 4 \\
66 \\
65 \\
7 \cdot 11 \times 10^{9}\end{array}$ & $\begin{array}{l}1: 3 \cdot 5 \\
76 \\
46 \\
6 \cdot 98 \times 10^{9}\end{array}$ & $\begin{array}{l}p<0.005 \\
p=0.06 \\
p<0.005 \\
N S\end{array}$ \\
\hline
\end{tabular}

Table 2 Positivity (\%) of selected HLA antigens in patients with $O R A$ and $Y R A$

\begin{tabular}{|c|c|c|c|}
\hline Positivity (\%) & ORA $(n=27)$ & $Y R A \quad(n=80)$ & Significance \\
\hline A25 & 0 & 0 & NS \\
\hline A26 & 15 & 13 & NS \\
\hline Cw3 & 41 & 49 & NS \\
\hline DR2 & 26 & 26 & NS \\
\hline DR3 & 0 & 0 & NS \\
\hline DR4 & 44 & 64 & $p=0.08$ \\
\hline
\end{tabular}

between ORA and YRA were found with any antigens, less DR4 positivity was observed in ORA $(44 \% v 64 \% ; \mathrm{p}=0.08)$.

Data on the initially involved joints were available for 66 patients with ORA and 77 with YRA. The involvement of various joints at the onset of the disease is listed in Table 3. Although the knee and shoulder joints were more often involved at the onset in ORA than in YRA, involvement of the fingers and forefoot were more common in YRA, and these differences were statistically significant, except for the shoulder. There was a tendency for the large joints rather than the small joints to be involved at the onset of the disease in patients with ORA.

Table 4 summarises the differences seen in ORA with OA and ORA without OA for various clinical measurements. Although there were no statistically significant differences in sex ratio, ESR, and HLA-DR4 positivity between the two groups, the lower RF positivity $(52 \% \vee 84 \% ; \mathrm{p}<0.01)$ and tendency towards early involvement of the knees or shoulders $(71 \% \vee 41 \% ; \mathrm{p}<0.025)$ were marked in ORA with OA.

Fourteen of 17 patients with ORA in whom the initially involved joint was the knee showed osteoarthritis on the roentgenograms taken at the onset of the disease. On the other hand, of 34 patients with

Table 3 Involvement of individual joints (\%) at onset in patients with $O R A$ and $Y R A$

\begin{tabular}{lccl}
\hline Joint* & $\begin{array}{l}\text { ORA } \\
(n=66)\end{array}$ & $\begin{array}{l}Y R A \\
(n=77)\end{array}$ & Significance \\
\hline Finger & 20 & 35 & $\mathrm{p}<0 \cdot 05$ \\
Forefoot & 3 & 13 & $\mathrm{p}<0 \cdot 01$ \\
Wrist & 20 & 17 & NS \\
Ankle & 11 & 8 & NS \\
Elbow & 6 & 7 & NS \\
Knee & 33 & 12 & $\mathrm{p}<0 \cdot 005$ \\
Shoulder & 21 & 10 & $\mathrm{p}<0 \cdot 1$ \\
Others & 8 & 3 & NS \\
\hline
\end{tabular}

* Multiple joints were counted in cases of polyarticular involvement at onset. 
Table 4 Influence of osteoarthritis (OA) on the clinical features of ORA

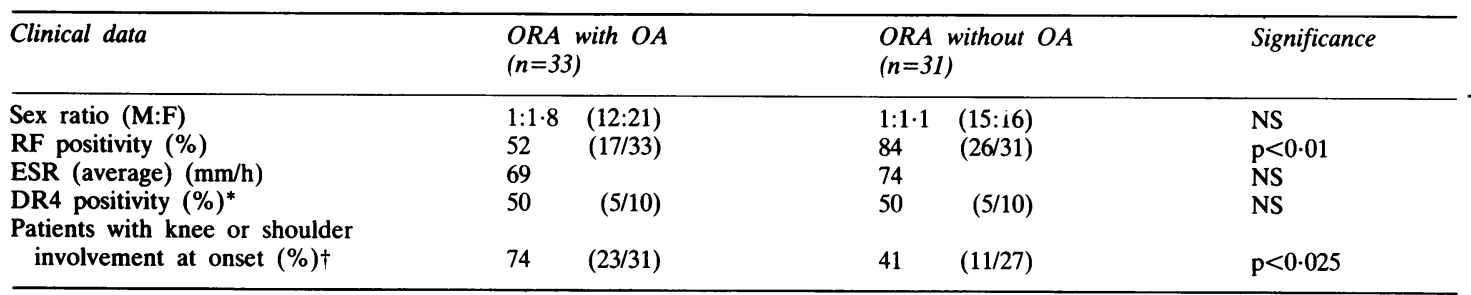

${ }^{*}$ Data were available for 10 patients with ORA and OA and for 10 patients with ORA but without OA.

†Data were available for 31 patients with ORA and OA and for 27 patients with ORA but without OA.

ORA in whom other joints were initially involved, only 13 had osteoarthritis in the knees on roentgenogram $(76 \% v 38 \% ; \mathrm{p}<0.005)$. The possibility that rheumatoid synovitis is likely to occur at its onset in large degenerative joints, particularly in the degenerative knee joint, was strongly suggested.

\section{Discussion}

In previous studies of ORA, the similar distribution between the sexes, ${ }^{236712}$ lower percentage of RF positivity, ${ }^{10}{ }^{12}$ and raised $\mathrm{ESR}^{14}$ have been described. The results of the present study agree with those of previous reports.

Terkeltaub et al reported an increase in HLADR4 and Cw3 in patients with ORA in comparison with normal controls. ${ }^{13}$ In our study the prevalence of DR4 positivity in ORA ( $44 \%)$ was less than that in YRA $(64 \%)$, but more than that reported for normal Japanese controls $(41 \%) .{ }^{15}$ Although there was no statistically significant difference, our data suggest that DR4 positivity may be related in some way to the age of onset of the disease.

In previous studies of joint involvement at the onset Dordick reported that half of the patients with ORA showed initial clinical manifestations in the shoulder ${ }^{3}$ and Oka and Kytila reported that fingers were most often affected at the onset in ORA. ${ }^{4}$ Of the patients with ORA in our study, the knees or shoulders were more often involved at the onset.

According to Dequeker et al osteoporosis and osteoarthritis are different diseases and not simply phenomena of aging. ${ }^{16}$ They are the systemic disease most likely to occur in the aged. Although few patients with YRA have osteoarthritis, many ORA patients do, and the clinical course of RA is likely to be modified by the existence of other systemic joint diseases. No report has been presented as to the effect of osteoarthritis on the clinical manifestations of patients with ORA.

In the present study less RF positivity and a proclivity towards large joint involvement at the onset were seen in ORA with osteoarthritis as opposed to ORA without osteoarthritis. It seems that for some patients the clinical features of ORA $\vec{N}$ are due to the presence of an associated degen- $\mathscr{O}$

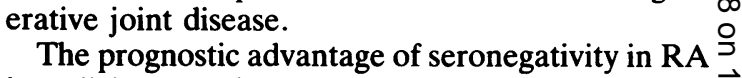
is well known. On the other hand, many authors

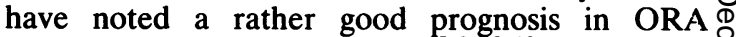
compared with RA in general. ${ }^{5-10} 12$ Taking into $\stackrel{\Phi}{7}$ account the lower percentage of RF positivity in 0 ORA with osteoarthritis in comparison with ORA $\stackrel{\Phi}{-}$ without osteoarthritis, as well as the osteoporosis $\overrightarrow{0}$ generally seen in severe RA, it is possible speculate that an osteoarthritis inducing factor m商 help to slow down the progression of RA.

The high prevalence of preceding osteophyte formation in the knees of patients with ORA, in whom the knee was the primary joint involved at the onset, demands a further explanation. Although knee complaints at the onset could be due to osteoarthritis which happened to precede the onset of RA, the fact that the interval between the occurrence of the knee complaints and subsequent involvement of other joints was less than three months in almost all patients shows that their knee $\frac{0}{0}$ complaints may be related to RA. It seems that large degenerative joints are susceptible to rheu- $\delta$ matoid synovitis at the onset of systemic RA.

In summary, we have postulated that some of the $ᄋ$ clinical features in ORA may be due to the relatively high prevalence of osteoarthritis in patients with ORA, and that the large degenerative joints may be particularly susceptible to rheumatoid inflammation 0 at the onset of RA. At the same time it is $\tilde{O}$ conceivable that the systemic effects of osteo- N arthritis may be negatively related to the progression of RA.

\footnotetext{
References

1 Schnell A. The clinical features of rheumatic infection in the old. Acta Med Scand 1941; 106: 345-50.

2 Cecil R L, Kammerer W H. Rheumatoid arthritis in the aged. Am J Med 1951; 10: 439-45.
} 
3 Dordick J R. Rheumatoid arthritis in the elderly. J Am Geriatr Soc 1956; 4: 588-91.

4 Oka M, Kytila J. Rheumatoid arthritis with the onset in old age. Acta Rheumatologica Scandinavica 1957; 3: 249-58.

5 Adler E. Rheumatoid arthritis in old age. Isr J Med Sci 1966; 2: 607-13.

6 Brown J W, Somes D A. The onset of rheumatoid arthritis in the aged. J Am Geriatr Soc 1967; 10: 873-81.

7 Moesmann G. Clinical features in subacute rheumatoid arthritis in old age. Acta Rheumatologica Scandinavica 1968; 14: 285-97.

8 Ehrlich G E, Katz W A, Cohen S A. Rheumatoid arthritis in the aged. Geriatrics 1970; 25: 103-13.

9 Corrigan A B, Robinson R G, Terenty T R, Dick-Smith J B, Walters D. Benign rheumatoid arthritis of the aged. Br Med J 1974; i: 444-6.

10 Deal C, Goldenberg D, Meenan R, et al. Elderly onset rheumatoid arthritis: A comparison with earlier onset, matched for disease duration [Abstract]. Arthritis Rheum 1981; 24 (suppl): 99.

11 Terkeltaub R, Esdaile J, Décary F, et al. Late onset rheumatoid arthritis [Abstract]. Arthritis Rheum 1981; 24 (suppl): 100.

12 Terkeltaub R, Esdaile J, Décary F, et al. A clinical study of older age onset rheumatoid arthritis with comparison to an early onset group. J Rheumatol 1983; 10: 418-24.

13 Terkeltaub R, Décary F, Esdaile J. An immunogenetic study of older age onset rheumatoid arthritis. J Rheumatol 1984; 11 : 147-9.

14 Kellgren J H, Lawrence J S. Rheumatism in miners. Br J Ind Med 1952; 9; 197-207.

15 Ohta N, Nishimura Y, Tanimoto K, et al. Association between HLA and Japanese patients with rheumatoid arthritis. Hum Immunol 1982; 5: 123-32.

16 Dequeker J, Goris P, Uytterhoeven R. Osteoporosis and osteoarthritis. JAMA 1983; 249: 1448-51. 